define three mutually exclusive sets of subgroups, and these three together constitute the totality of the cyclic subgroups of $G_{\frac{1}{2}\left(p^{2}-1\right)}$. Their number is seen to be $p^{2}+p+1$. The order of the subgroup is $p$ when $\lambda \equiv 0$, when $4 \mu \nu+1 \equiv 0$, and in the second pair; the order is $\frac{1}{2}(p-1)$ when $\lambda$ or $4 \mu \nu+1$ is a quadratic residue of $p$; the order is $\frac{1}{2}(p+1)$ when $\lambda$ or $4 \mu \nu+1$ is a non-residue of $p$.

This explicit form of definition seems to be new, and on account of its simplicity may be of interest.

NORTHWESTERN UNIVERSITY,

September, 1904 .

\title{
EXTENSION OF A THEOREM DUE TO SYLOW.
}

BY PROFESSOR G. A. MILLER.

(Read before Section A of the American Association for the Advancement of Science, Philadelphia, December 29, 1904.)

Every group $G$ of order $p^{m}, p$ being any prime number, contains at least $p$ invariant operators. This fundamental theorem, due to Sylow, ${ }^{*}$ is included in the following: Every non-abelian group of order $p^{m}$ contains at least $p$ invariant commutator operators, and its commutator quotient group $\dagger$ is always non-cyclic. In this connection it seems desirable to prove the following closely related theorems : It is possible to construct a non-abelian group having any arbitrary abelian group as a commutator quotient group. Every non-cyclic abelian group of order $p^{a}$ is the commutator quotient group of some nonabelian group of order $p^{m}$.

The first part of the theorem in italics may be proved as follows: Let $H$ represent the subgroup of $G$ which is composed of its $p^{\beta}$ invariant operators and let $H_{1}$ represent an invariant subgroup of order $p^{\beta+1}$ which includes $H$. $\$$ Any operator $s$ of $G$ which is not commutative with all the operators of $H_{1}$ transforms $H_{1}$ into a simple isomorphism with itself such that each of the operators of $H$ corresponds to itself. Since $H_{1}$ is

\footnotetext{
* Sylow, Math. Annalen, vol. 5 (1872), p. 584.

+ It seems convenient to speak of the quotient group corresponding to the commutator subgroup as the commutator quotient group.

$\ddagger$ Every invariant subgroup of a group of order $p^{m}$ is included in a larger invariant subgroup of arbitrary order less than $p^{m}$.
} 
abelian, its remaining operators are transformed into themselves multiplied by operators of order $p$ contained in $H . *$ These $p-1$ commutators of order $p$ are invariant under $G$ by hypothesis.

To prove that the commutator quotient group of $G$ is noncyclic it is only necessary to observe that $G$ contains more than one subgroup of order $p^{m-1} \dagger$ and that the operators which are common to all the subgroups of this order constitute a characteristic subgroup of $G$ with respect to which the quotient group is abelian and of type $(1,1,1, \ldots)+$. As this quotient group has a $(1, k)$ isomorphism with the commutator quotient group, $\S$ and is of composite order, the latter is non-cyclic. This completes the proof of the theorem in italics.

Since the commutator quotient group of the direct product of two groups is the direct product of the commutator quotient groups of these two groups, the proof that we can construct a non-abelian group whose commutator quotient group is the cyclic group of order $p^{a}$, $\alpha$ being arbitrary, involves the proof of the theorem that it is possible to construct a non-abelian group whose commutator quotient group is an arbitrary abelian group. We proceed to prove this theorem.

It is well known that the infinite arithmetic series

$$
1,1+p^{a}, 1+2 p^{a}, 1+3 p^{a}, \cdots
$$

involves an infinite number of prime numbers. Let $p_{1}$ represent one of these primes. Since $p_{1}-1$ is divisible by $p^{\alpha}$ there are numbers which belong to exponent $p^{a} \bmod p_{1}$. In other words, there are groups whose commutator quotient group is the cyclic group of order $p^{a}$. This proves the theorem in question. It may be remarked that every transitive substitution group of degree $p$ has a cyclic commutator quotient group. $\|$

The fact that every possible non-cyclic abelian group of order $p^{a}$ is the commutator quotient group of some non-abelian group of order $p^{m}$ may be proved as follows: If the abelian group of order $p^{a}$ has only two invariants $p^{\alpha_{1}}, p^{\alpha_{2}}\left(\alpha_{1} \equiv \alpha_{2}\right)$, we

\footnotetext{
* Bulletrin, vol. 6 (1900), p. 337. Annals of Mathematics, ser. 2, vol. 3 (1902), p. 180 .

† Burnside, Theory of groups, 1897, pp. 73, 75.

$\ddagger$ Bauer, Nouvelles Ann. de Math., vol. 19 (1900), p. 509 .

Q Quar. Jour. of Math., vol. 28 (1896), p. 268.

II Bulletin, vol. 4 (1898), p. 141. Cf. Sylow, Math. Annalen, vol. 5 (1872), p. 594 .
} 
let $t$ represent any operator of order $p$ which transforms the generator $s$ of a cyclic group of order $p^{a_{1}+1}$ into its $p^{\alpha_{1}}+1$ power. The commutator quotient group of the group generated by $s$ and $t$ is clearly of type $\left(\alpha_{1}, 1\right)$. Let $t^{\prime}$ represent any operator of order $p^{\alpha_{2}}$ which is independent of $s$ and $t$. The operators $s$ and $t t^{\prime}$ will then generate the required non-abelian group. By forming the direct product of this non-abelian group and some abelian group any additional invariants may be introduced into the commutator quotient group. Hence the theorem is proved.

STANFORD UNIVERSITY, December, 1904.

\section{NOTE ON ISOTHERMAL CURVES AND ONE- PARAMETER GROUPS OF CONFORMAL TRANSFORMATIONS IN THE PLANE.}

BY PROFESSOR C. L. BOUTON.

IN the January number of the Bulletin, page 180, Mr. J. E. Wright integrated a certain differential equation by determining a continuous group which the equation admits. In solving the problem Mr. Wright determines a group of conformal transformations with given path curves. In this connection, it is an obvious problem to find the necessary and sufficient conditions under which a conformal group with given path curves shall exist. The solution of this problem is given in the following theorem:

Theorem. - A one-parameter group of conformal transformations with given path curves exists when and only when the given curves form an isothermal family.

Although this theorem seems very obvious the writer cannot find it in print, and, therefore, gives two easy proofs for it.

I. Let $U f=\xi p+\eta q$ be the symbol of the infinitesimal transformation of the group. Since the group is to be conformal, we must have $\xi+i \eta=\phi(x+i y)$. The differential equation of the path curves is $\eta d x-\xi d y=0$. From this equation we have

$$
\frac{d x+i d y}{\xi+i \eta}=\frac{d x-i d y}{\xi-i \eta}
$$

